\title{
A ROD-SHAPED VIBRO TOUCH SENSOR USING PZT THIN FILM
}

\author{
Takefumi KANDA, Takeshi MORITA, Minoru KUROSAWA, Toshiro HIGUCHI \\ Department of Precision Machinery Engineering, Graduate School of Engineering, \\ The University of Tokyo, 7-3-1 Hongo, Bunkyo-ku, Tokyo 113-8656, JAPAN \\ Tel +81-3-3812-2111 ext.6449, Fax +81-3-5800-6968 \\ E-mail: kanda@intellect.pe.u-tokyo.ac.jp
}

\begin{abstract}
ABSTRUCT
We have fabricated a probe sensor. This sensor is for high-precision coordinate measuring machines, surface roughness measuring tools, or scanning probe microscopes (SPM). This sensor consists of a rod vibrating in the axial direction. The longitudinal vibration was excited and also detected by PZT thin film. The PZT thin film was fabricated by a hydrothermal method. The hydrothermal method uses the reaction process in hot and high pressure aqueous solutions. We made $27.8 \mathrm{~mm}$ long sensor. Its resonance frequency was $116 \mathrm{kHz}$. The sensitivity and resolution were evaluated by experiments. We succeeded in oscillating the rod and detecting the contact.
\end{abstract}

\section{INTRODUCTION}

Recently, the demand for prove-sensors is increasing. This is because MEMS technology has made progress. It requires high-precision coordinate-measuring machines. A touch sensor which is vibrating longitudinally has been proposed as a probe of such measuring machines ${ }^{1]}$. If the probe is miniaturized, surface roughness will also be measured, then it will be applicable to scanning probe microscopes (SPM).

In most of tactile sensors and SPM probes, bending vibration of cantilevers are used. However, the $Q$ value of cantilevers cannot be so high under millimeter scale. It is because the damping ratio by air viscosity increases. The sensitivity of vibro probe sensors depends on the mechanical $Q$ factor. The high damping ratio by air viscosity make the $Q$ value lower. However, the longitudinal vibrating rod is not so influenced by damping ratio by air viscosity. Therefore it is possible to maintain high $Q$ value. So it is consider that longitudinal vibration sensor can obtain higher sensitivity and resolution than cantilevers in micro scale. In addition, because of the small damping ratio, the longitudinal vibration sensor is useful in liquid.

Piezo electric materials are used to oscillate probes and detect the contact. To miniaturize the prove sensor, piezoelectric thin films are useful. By using thin films, the piezo material and rod can be connected without any adhesive or bolts.

There are several methods to deposit a piezoelectric thin film. However, it is difficult to get enough force for oscillators. We deposited a lead zirconate titanate (PZT) thin film by a hydrothermal method ${ }^{21}$. With this method, thickness of the film is about $10 \mu \mathrm{m}$. Using this method, PZT thin films are formed on three-dimensional complex-shaped bodies. So we can easily miniaturize the oscillator. A micro ultrasonic motor has been fabricated by using this method ${ }^{3]}$.

We used PZT thin film by the hydrothermal method for oscillating longitudinal vibration and detecting the contact. It may be possible to make much smaller micro-sensors by the hydrothermal method.

\section{STRUCTURE AND PRINCIPLE}

\section{Principle}

As shown in Fig. 1, the sensor is a rod-shaped vibrator. The sensor vibrator has half wave length of longitudinal vibration mode. An exponential horn which enlarges the vibration amplitude increases sensitivity. When the tipend of the horn takes into physical contact with a workpiece, the resonance frequency of the vibrator is shifted. By detecting the frequency shift, the contact is sensed.

For the theoretical consideration, this vibro sensor can be modeled as shown in Fig. $2^{4 !}$. The sensor fixed in the

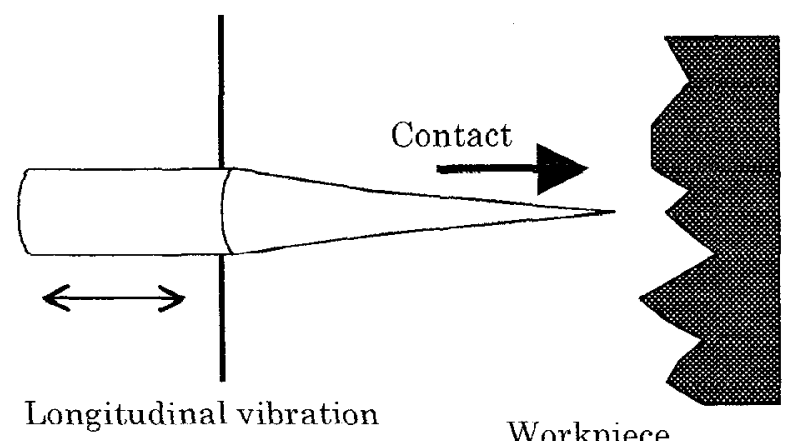

Fig. 1 Sketch of the measurement of the object shape by the vibro touch sensor. 


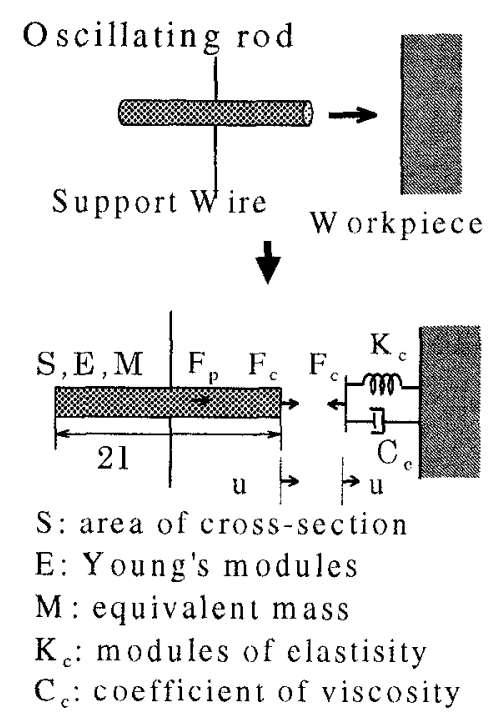

Fig. 2 Model of the vibro touch sensor.

node of the longitudinal vibration. Then horn-part and cylindrical part of the oscillator will be equally vibrating: both parts are quarter wave length. From the equation of motion, the resonance angular frequency is expressed by

$$
\omega=\frac{1}{m} \sqrt{\left(\frac{S E}{l}+K_{c}\right)^{2}-\frac{C_{c}^{2}}{2}}
$$

When there is no contact, $K_{c}=C_{c}=0$. When the contact take place, these variables change and the resonance frequency also shifts.

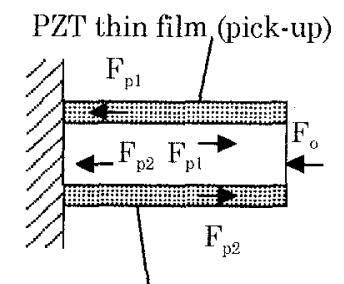

PZT thin film (pick-up)

(a)

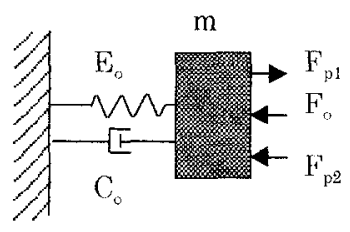

(b)
Fig. 3 Model of Ti base and PZT thin film: The Oscillating rod is supported at the node of the vibration. Then we can consider the behavior of the rod by using half part of the rod.

In the model at Fig.3, the sensor consists of PZT thin film for driving, the base material (titanium rod), and PZT thin film for pick-up. By considering the interaction between these parts, the equation of motion is written in the form

$$
m \frac{d^{2} u}{d t^{2}}+C_{o} \frac{d u}{d t}+S_{o} E_{o} \frac{u}{l}+F_{o}-F_{p 1}+F_{p 2}=0
$$

From this equation, the equivalent circuit of this sensor is obtained as shown in Fig.4. In this circuit, the pick-up voltage of the sensor is expressed in

$$
\begin{aligned}
V_{\text {out }}=-\frac{A_{1} A_{2}}{\omega C_{d 2}} & \frac{V_{\text {in }}}{R^{2}+\left(\omega L-\frac{1}{\omega C}-\frac{A_{2}^{2}}{\omega C_{d 2}}\right)^{2}} \\
& \cdot\left\{\left(\omega L-\frac{1}{\omega C}-\frac{A_{2}^{2}}{\omega C_{d 2}}\right)+j R\right\}
\end{aligned}
$$

At resonance angular frequency $\left(\omega=\omega_{s}\right)$, pick-up voltage is expressed by,

$$
V_{\text {out }}=\frac{A_{1} A_{2}}{j \omega C_{d 2}+A_{2}^{2}} V_{\text {in }}
$$

Therefore, by detecting the pick-up voltage, the vibration can be detected. Then the contact can be sensed from the pick-up voltage of the sensor.

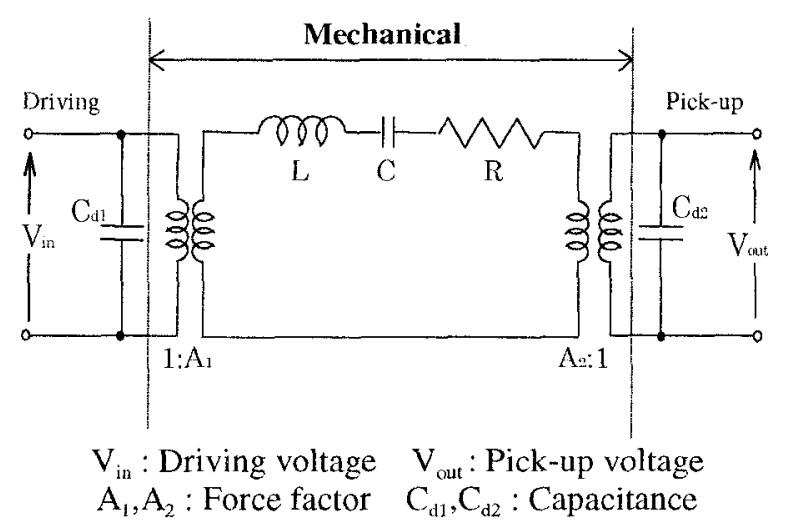

Fig. 4 Equivalent circuit of the sensor.

In the theoretical consideration here, we don't take into account any near-field effect. However, hydrodynamic friction is considered to have some effects ${ }^{51}$. So, to discuss the behavior of the oscillator's vibration near the surface, we have to consider the hydrodynamic condition.

\section{Structure}

The structure of the vibro sensor is shown in Figs.5, 6, and 7. Its length and thick diameter are $27.7 \mathrm{~mm}$ and 2.4 $\mathrm{mm}$. The core material of this sensor is titanium. The titanium is covered with PZT thin film. The thickness of the film is $10 \mu \mathrm{m}$. On the surface, there exists four 
electrodes for piezoelectric drive and pick-up.

The rod vibrates in the axial direction. One end of the rod was formed into an exponential horn. The horn enlarged the vibration amplitude. The magnification ratio was 4.8 .

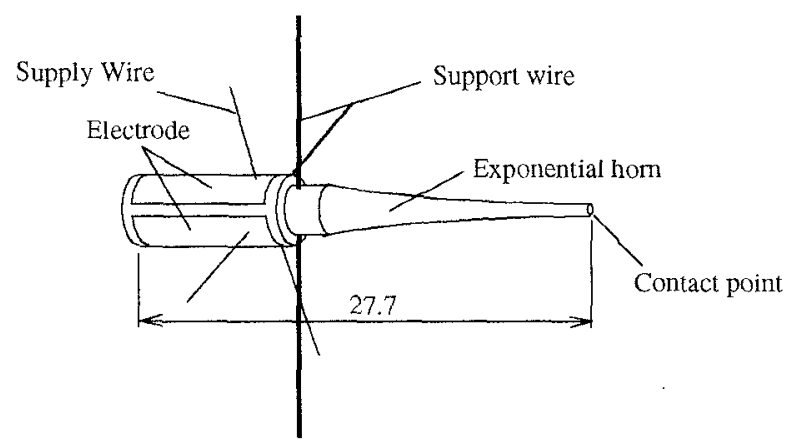

Fig. 5 Schematic shape of the sensor.

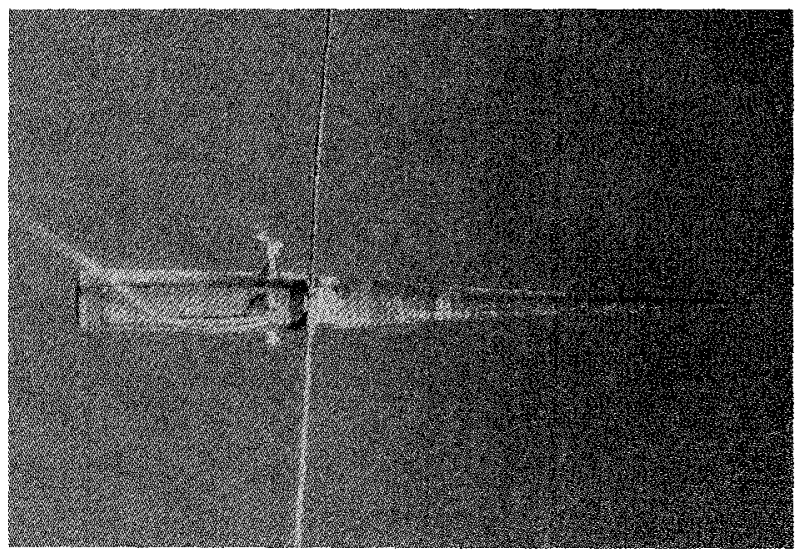

Fig. 6 Photograph of the sensor.

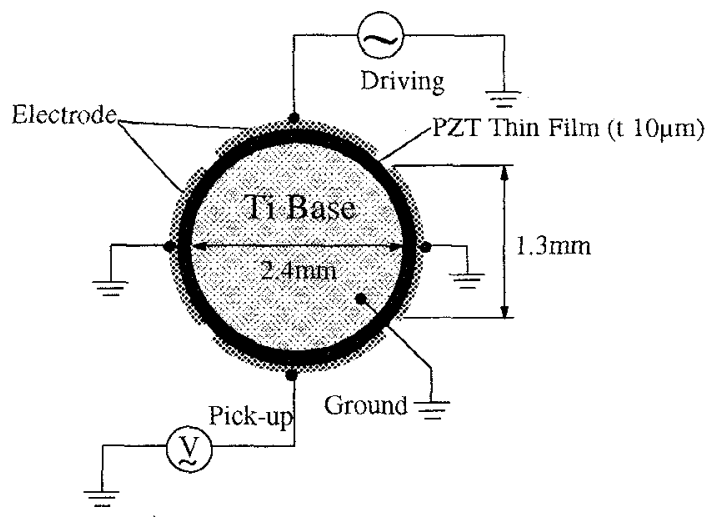

Fig. 7 Cross section of the sensor.

\section{Hydrothermal method}

PZT thin film formed on this sensor is fabricated by the hydrothermal method ${ }^{21}$. By using this fabrication method, we can obtain the polarized PZT film on titanium base. Figure 8 shows the image of this reaction process. This reaction is carried out in a solution which contains $\mathrm{Pb}^{2+}, \mathrm{Zr}^{2+}$ and $\mathrm{Ti}^{4+}$. This solution and base material is kept at high temperature and high pressure.

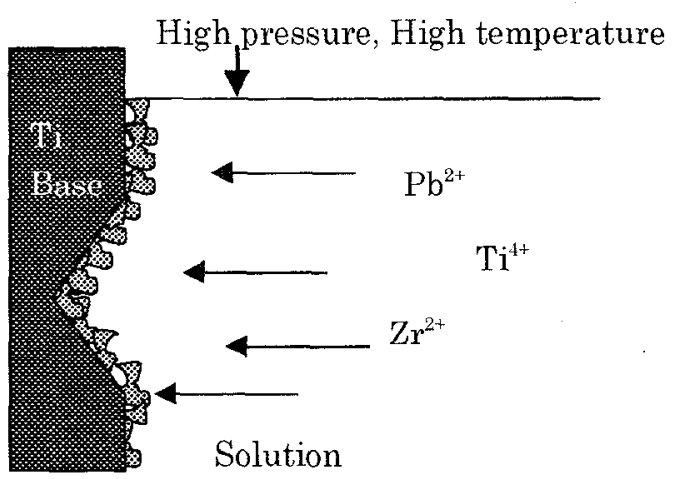

Fig. 8 Image of the hydrothermal method.

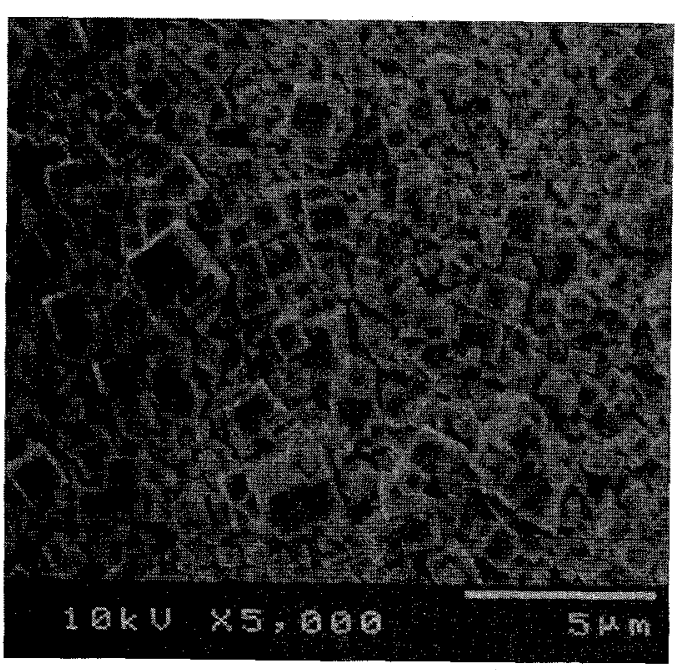

Fig. 9 SEM photo of the film deposited by the single process hydrothermal method.

We used a PZT thin film formed by a single process hydrothermal method ${ }^{6]}$. The previous hydrothermal method consists of two processes, namely, the nucleation process and the crystal growth process. Between these two processes, there are difference in the conditions for the concentration of solutions and the reaction temperatures. It brought that it is difficult to control the characteristics of films. Moreover, the film fabricated by the previous method contained lead zirconate (PZ) layer. $\mathrm{PZ}$ is antiferroelectric substance, so that the piezoelectric effect was diminished.

The single process hydrothermal method simplified the process. The reaction conditions are shown in Table 1 . Figure 9 shows a scanning electro microscope (SEM) photograph of the surface of the film deposited by the 
single process hydrothermal method. This reaction was carried out three times, so that the thickness of the film was $10 \mu \mathrm{m}$. In this method, pretreatment process is omitted. So the chemical composition will be easily controlled. In PZT, the piezoelectric effect depends on the $\mathrm{Zr} /(\mathrm{Ti}+\mathrm{Zr})$ ratio. We have succeeded in controlling the chemical composition by changing the $\mathrm{Zr} /(\mathrm{Ti}+\mathrm{Zr})$ ratio in the solution.

Table 1 The reaction conditions of the single process hydrothermal method.

\begin{tabular}{lll}
\hline $\mathrm{Pb}\left(\mathrm{NO}_{3}\right)_{2}$ & $1.204[\mathrm{~g}]$ & Melted in $5.5[\mathrm{ml}] \mathrm{H}_{2} \mathrm{O}$ \\
$\mathrm{ZrOCl}_{2} 8 \mathrm{H}_{2} \mathrm{O}$ & $0.507[\mathrm{~g}]$ & Melted in $2[\mathrm{ml}] \mathrm{H}_{2} \mathrm{O}$ \\
$\mathrm{TiCl}_{4}$ & $0.71[\mathrm{~mol} / \mathrm{l}]$ & $1.78[\mathrm{ml}]$ \\
$\mathrm{KOH}$ & $2.69[\mathrm{~g}]$ & Melted in $11[\mathrm{ml}] \mathrm{H}_{2} \mathrm{O}$ \\
\hline Reaction temperature & 140 degrees \\
\hline Reaction pressure & $3.6[\mathrm{~atm}]$ \\
\hline Reaction period & $24[\mathrm{~h}] / 1$ process \\
\hline
\end{tabular}

\section{EXPERIMENTAL RESULTS}

\section{Vibrational amplitude and pick-up}

To estimate the resonance frequency and the longitudinal vibration amplitude, we measured the vibrational velocity at the horn-tip and the pick-up voltage. Figure 10 shows the shame of the measurement. We used one electrode for driving, and another one for pick-up. These two electrodes exists on the opposite side of the cylinder. Other electrodes were connected to the ground. The driving voltage was kept at $5 \mathrm{~V}_{\mathrm{p}-\mathrm{p}}$. The vibration velocity at the horn-tip was measured by using a laser Doppler vibrometer. The output voltage from the pick-up electrode was also measured by using a lock-in amplifier.

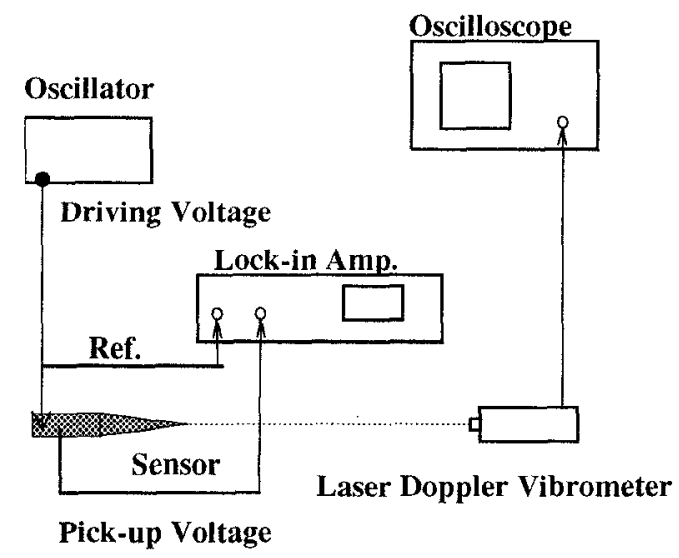

Fig. 10 Set-up for the measurement of the vibrational amplitude and pick-up voltage.

The results are shown in Fig.11. As shown in the graph, the resonance frequency was $115.24 \mathrm{kHz}$. The maximum value of amplitude was $7.9 \mathrm{~nm}_{\mathrm{o}-\mathrm{p}}$. The pick- up voltage also had its peak at the resonance. Hence it is possible to detect the shift of the resonance frequency by measuring the pick-up voltage. The mechanical $Q$ factor was 610 .

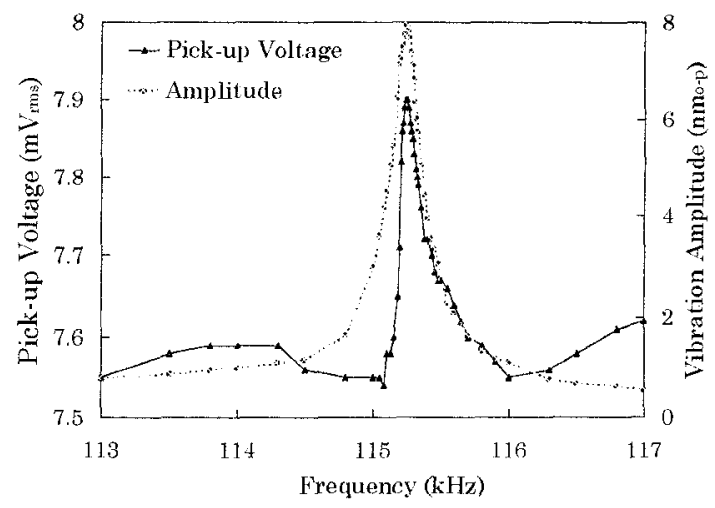

Fig. 11 Relationship between the amplitude at the horn-tip and pick-up voltage.

The output voltage contained offset as shown in Fig.12. This offset was considered due to the leakage from driving voltage. At the resonance, the offset was $7.6 \mathrm{mV}$ rms while the signal from the vibration was only $0.3 \mathrm{mV}_{\mathrm{rms}}$. So the offset was not negligible error. If the offset is diminished or the vibrational signal improves, the error will decrease.

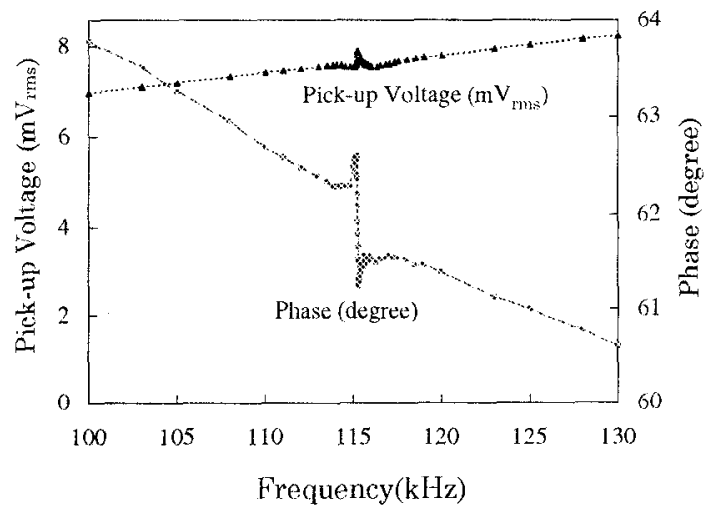

Fig. 12 Relationship between pick-up voltage and phase.

\section{Detection of the contact}

The shift of the resonance frequency due to the contact of the horn-tip to an object was measured from the pickup signal. We used the measurement set-up as shown in Fig. 13 for the test to detect the contact by this sensor, and to determine its resolution. We used a linear stage driven by stepping motor for the large scale displacement and a multi-layer type PZT element for the small scale displacement. The displacement 
corresponding to 1 step of the motor was $1 \mu \mathrm{m}$ of the linear stage. The displacement by the PZT element was $66 \mathrm{~nm}$ per 1V D.C. The displacement of the PZT element was measured by using a capacitance displacement meter. Onto the layer type PZT element, we fixed an aluminum sheet as a workpiece. Then the test to detect the contact was carried out by bringing the workpiece close to the horn-end of the sensor. To determine contacts, we also measured the electric resistance between the workpiece and the sensor.

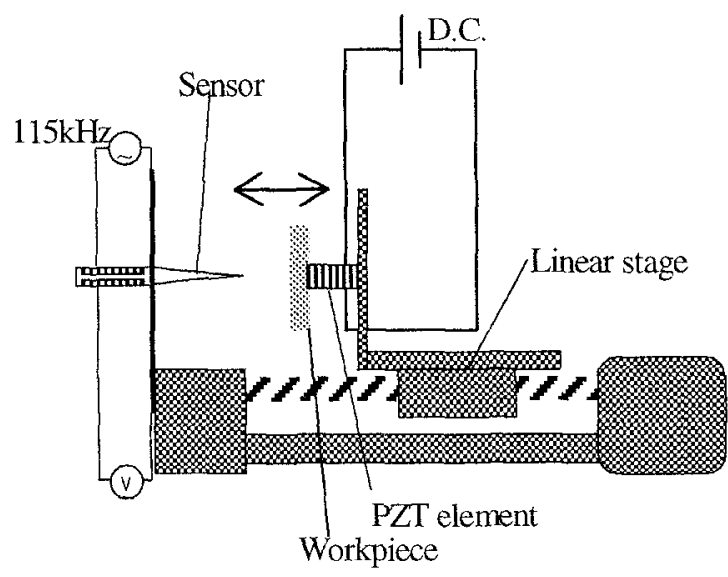

Fig. 13 Set-up for the measurement of the detection of the contact.

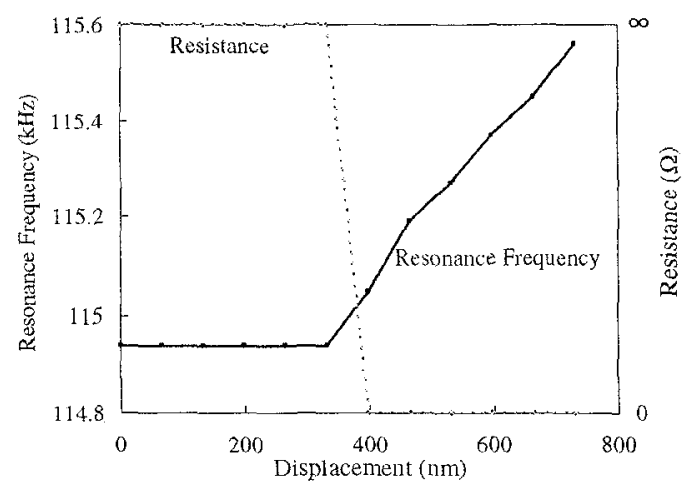

Fig. 14 Resonance frequency shift by the contact.

The results are shown in Fig. 14. The graph indicates the relationship between the resonance frequency and the displacement of the workpiece. The resonance frequency was sensed from the pick-up voltage which gave the maximum value. Contact or non-contact condition was also detected by the resistance between the horn-tip and the workpiece. The shift of the resistance and that of resonance frequency begins at the same displacement. So the contact can be detected by monitoring the pick-up voltage. In this measurement, the workpiece moved every $66 \mathrm{~nm}$. Therefore the resolution of the contact detection was higher than $66 \mathrm{~nm}$

The contact will be detected not only by the shift of the resonance frequency, but also by monitoring the decrease of the oscillator amplitude. We measured the vibration amplitude when the contact took place. In addition to measurement set-up in the Fig.13, the vibration amplitude at the cylindrical end was measured with a laser Doppler vibrometer. The driving voltage frequency was kept at the resonance frequency at non contact.

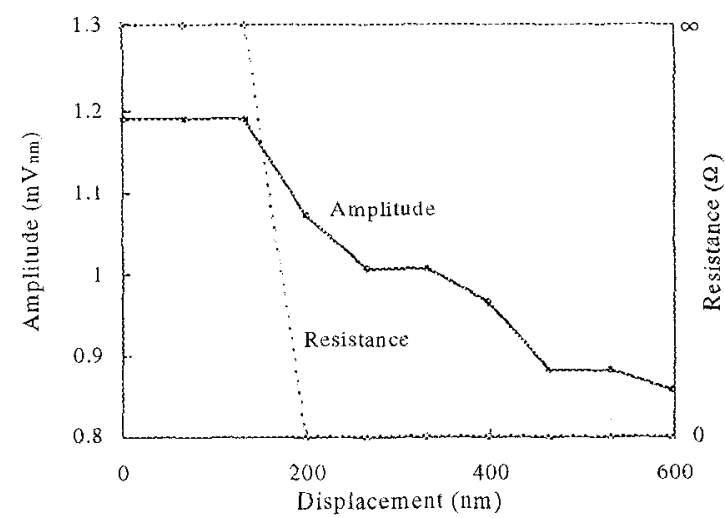

Fig. 15 Vibration amplitude shift by the contact.

Figure 15 indicates the relationship between the shift of the vibration amplitude and the displacement. The decrease of the amplitude appeared at the displacement where the resistance changed to null. So when the contact takes place, the vibration amplitude decreased. As the vibration amplitude decreases, the pick-up voltage decreases. When the frequency of the driving voltage is fixed, the contact can be detected by monitoring the pick-up voltage.

\section{DISCUSSIONS}

The constants of the equivalent circuit in Fig.4 were calculated using the horn-tip amplitude, equivalent mass, and the mechanical $Q$ value.

Table 2 Constants of the equivalent circuit.

\begin{tabular}{lll}
\hline $\mathrm{L}$ & Equivalent mass & $1.0 \times 10^{-4}[\mathrm{H}]$ \\
$\mathrm{C}$ & Equivalent capacitance & $1.9 \times 10^{-8}[\mathrm{~F}]$ \\
$\mathrm{R}$ & Equivalent resistance & $1.2 \times 10^{-4}[\Omega]$ \\
$\mathrm{C}_{\mathrm{d} 1}$ & Capacitance & $1.3 \times 10^{-8}[\mathrm{~F}]$ \\
$\mathrm{A} 1$ & Force factor & $8.6 \times 10^{-5}[\mathrm{~N} / \mathrm{V}]$ \\
\hline
\end{tabular}

As shown in Fig,4, electrical parts, for the driving and 
the pick-up, are symmetric respect to the mechanical part. So, in the equivalent circuit, the relations of $C_{d I}=$ $C_{d 2}$, and $A_{1}=A_{2}$ should hold. When driving voltage $V_{\text {in }}$ is $5.0 \mathrm{~V}_{\mathrm{p}-\mathrm{p}}$, the pick-up voltage $V_{\text {out }}$, is predicted to be $1.2 \times 10^{-5}\left[\mathrm{~V}_{\text {rms }}\right]$. However, from the measurement, $V_{\text {out }}$ was $3.0 \times 10^{-4}[\mathrm{~V}]$. The main cause for this error is thought that the pick-up voltage contains the offset. This offset is defined as the value taken away actual signal of vibration from the pick-up voltage. The offset is thought to be due to the leakage of driving voltage between electrodes or supply wires. From the measurement, the offset was approximately 23 times as much as the actual signal of the vibration.

To reduce the influence of the leakage signal, the proportion of PZT to $\mathrm{Ti}$ base have to be increased. If the proportion increases, an influence by the internal friction of $\mathrm{Ti}$ base material decreases. Therefore the pick-up voltage will increase, and the driving voltage for the vibration will be reduced. So the sensitivity of the sensor can be improved. As a result, the actual signal of the vibration will increase against the leakage signal.

As the oscillator miniaturized, the proportion increases. This is because the volume of PZT depends on the surface area of $\mathrm{Ti}$ base. In addition, if the oscillator has a flat configuration as shown in Fig.16, the proportion increases.

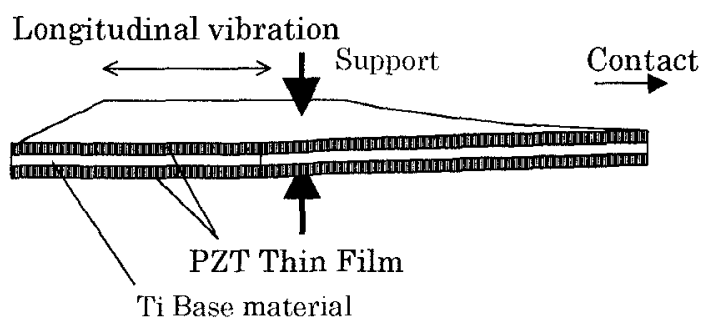

Fig. 16 Flat type oscillator.

\section{CONCLUSIONS}

We succeeded in oscillating the rod-shaped sensor and detecting the contact by using PZT thin film fabricated by the hydrothermal method. The resolution for detecting the contact was higher than $66 \mathrm{~nm}$. The hornend-amplitude at the resonance was $7.9 \mathrm{~nm}_{\text {o-p. }}$. So the resolution of the sensor would be up to $10 \mathrm{~nm}$.

The pick-up voltage included offset. Then it influenced the sensitivity and resolution. By improving the structure of the sensor, the influence of the offset will decrease.

It will be possible to achieve higher sensitivity and resolution by miniaturizing the sensor and making the resonance frequency higher.

\section{REFERENCES}

[1] S.M.Harb, M.Vidic "Resonator-based touchsensitive probe", Sensors and Actuators A 50 pp2329, (1995).

[2] K.Shimomura, T.Tsurumi, Y.Ohba and M.Daimom: "Preparation of Lead Zirconate Titanate Thin Film by Hydrothermal Method", Jpn. J. Appl. Phys, Vol.30, No.9B, pp.2174, (1991).

[3] T.Morita, M.Kurosawa and T.Higuchi: "A Micro Ultrasonic Motor Using Bending Cylindrical Transducer Based on PZT Thin Film" Sensors \& Actuators: A 50, pp75-80, (1996).

[4] M.Nishimura, K.Hidaka, M.Teraguti: Proceedings of annual spring meeting of the JSPE, p.765, (1994), (in Japaneese).

[5] M.Giousouf, M.Weinmann, W.Scheerer, F.Assmus, W.v.Munch: "Dynamic behaviour of a Quartz extensional-mode non-tactile profile sensor", Sensors and Actuators A 61 pp287-292, (1997).

[6] T.Morita, T.Kanda, M.Kurosawa and T.Higuchi: "Single Process to Deposit Lead Zirconate Titanate (PZT) Thin Film by a Hydrothermal method", Jpn. J. Appl. Phys, Vol.36, No.5B, pp2998-2999, (1997) 